\title{
An Effective $z$-Stretching Method for Paraxial Light Beam Propagation Simulations ${ }^{1}$
}

\author{
Leonel Gonzalez ${ }^{\dagger}$, Shekhar Guha ${ }^{\dagger}$, James W. Rogers ${ }^{2 \natural}$ and Qin Sheng, ${ }^{b, \natural}$ \\ ${ }^{\dagger}$ Materials \& Manufacturing Directorate, Air Force Research Laboratory \\ 2977 Hobson Way, Wright-Patterson AFB, OH 45433-7702, USA \\ ${ }^{b}$ Center for Astrophysics, Space Physics \& Engineering Research \\ ${ }^{\natural}$ Department of Mathematics \\ Baylor University, Waco, TX 76798-7328, USA
}

\begin{abstract}
A $z$-stretching finite difference method is established for simulating the paraxial light beam propagation through a lens in a cylindrically symmetric domain. By introducing proper domain transformations, we solve corresponding difference approximations on a uniform grid in the computational space for great efficiency. A specialized matrix analysis method is constructed to study the numerical stability. Interesting computational results are presented.
\end{abstract}

Keywords. Light beam propagation, interface or discontinuous surface, domain transformation, consistency, stability, uniform and nonuniform grids, approximations

AMS (MOS) Subject Classification: 65M06, 65M50, 65Z05, 78A15, 78M20

\section{Introduction}

In order to reduce the computational complexity of light beam propagation simulation, a number of approximations are typically employed. We take advantage of these approximation techniques for the paraxial case to derive an efficient and robust method that allows the application of conventional finite difference schemes on a uniform grid in the computational space, despite the difficulty of an interface present in the domain.

From Maxwell's field equations describing the behavior of monochromatic light, we obtain the time-dependent Helmholtz equation,

$$
\nabla^{2} E-\frac{1}{c^{2}} \frac{\partial^{2} E}{\partial t^{2}}=0
$$

where $E=E(x, y, z, t)$ is the electric field intensity, $\nabla^{2}$ is the Laplacian operator, and $c$ is the phase velocity, or speed of light in a particular medium.

\footnotetext{
${ }^{1}$ The third and fourth authors are supported in part by a research grant (No. AFGD-035$75 \mathrm{CS}$ ) from the Air Force Research Laboratory and General Dynamics. The fourth author is also supported in part by ASEE-SFFP Awards from the U.S. Air Force.

${ }^{2}$ Principal and corresponding author. Email address: James_W_Rogers@baylor.edu
} 
Let $E$ be the field intensity of a monochromatic plane wave of the form

$$
E(x, y, z, t)=U(x, y, z) e^{i 2 \pi \nu t}
$$

where $\nu$ is the frequency of the light. Then from (1.1) we acquire the timeindependent Helmholtz equation

$$
\left(\nabla^{2}+\kappa^{2}\right) U(x, y, z)=0
$$

where $\kappa=2 \pi \nu / c=2 \pi / \lambda$ is referred as the wave number, and $\lambda=c / \nu$ is referred as the wavelength. Functions $|U(x, y, z)|$ and $\arg (U(x, y, z))$ are the amplitude and phase of the wave, respectively. Assume that the $z$ is the direction of the beam propagation. We may further consider the wave function with a complex amplitude, that is,

$$
U(x, y, z)=u(x, y, z) e^{-i \kappa z}
$$

where $u$ is called a complex envelope. A paraxial wave becomes realistic if the variation of $u$ is slow in the $z$-direction.

Substitute (1.4) into (1.3) to yield

$$
\nabla_{T}^{2} u(x, y, z)-2 i k \frac{\partial u(x, y, z)}{\partial z}+\frac{\partial^{2} u(x, y, z)}{\partial z^{2}}=0
$$

where

$$
\nabla_{T}^{2}=\frac{\partial^{2}}{\partial x^{2}}+\frac{\partial^{2}}{\partial y^{2}}
$$

is the transverse Laplacian operator. In a paraxial case, we may assume that within a wavelength of the propagation distance, the change in $u$ is sufficiently small compared to $|u|[12]$. Thus,

$$
\left|\frac{\partial^{2} u}{\partial z^{2}}\right| \ll\left|\kappa^{2} u\right|
$$

which indicates that

$$
\frac{\partial^{2} u}{\partial z^{2}} \approx 0
$$

Therefore we arrive at an approximation of (1.5),

$$
\nabla_{T}^{2} u(x, y, z)-2 i \kappa \frac{\partial u(x, y, z)}{\partial z}=0
$$

which is called the slowly varying envelope approximation of the Helmholtz equation $[1,6,13]$. 
Under the transformation $r=\sqrt{x^{2}+y^{2}}$ and $\phi=\arctan (y / x),(1.6)$ can be reformulated to

$$
\left(\frac{1}{r} \frac{\partial}{\partial r}+\frac{\partial^{2}}{\partial r^{2}}+\frac{1}{r^{2}} \frac{\partial^{2}}{\partial \phi^{2}}-2 i \kappa \frac{\partial}{\partial z}\right) u(r, z)=0
$$

The equation (1.7) has been utilized frequently in laser beam propagation simulations in the past decades $[6,7,16]$.

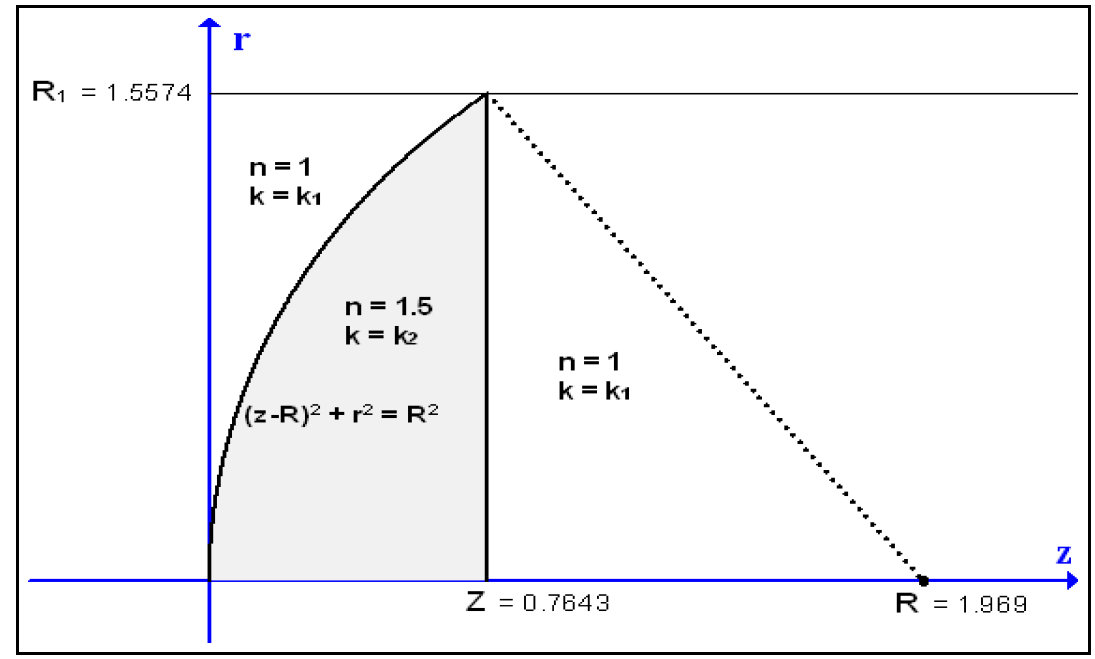

Figure 1.1. An illustration of the leans area [15].

In this paper, we shall consider a cylindrically symmetric domain for spherical lens environments. For the situation we may assume [6] that

$$
\frac{\partial^{2} u}{\partial \phi^{2}} \equiv 0
$$

and (1.7) can be simplified to yield

$$
2 i \kappa \frac{\partial u}{\partial z}(r, z)=\frac{\partial^{2} u}{\partial r^{2}}(r, z)+\frac{1}{r} \frac{\partial u}{\partial r}(r, z), \quad 0 \leq r \leq r_{0} \ll \infty .
$$

We assume that the wavelength of light, $\lambda$, is $9.449 \mu \mathrm{m}$ and that light is incident from air (refractive index $n_{1}=1$ ) into glen (refractive index $n_{2}=1.5$ ) so that we may adopt the following wave numbers [6]

$$
\kappa(r, z)= \begin{cases}\kappa_{0}=\frac{2 \pi n_{1}}{\lambda} \approx \frac{2}{3} \times 9.97543 \times 10^{3} \mathrm{~cm}^{-1}, & \text { in medium one; } \\ \kappa_{1}=\frac{2 \pi n_{2}}{\lambda} \approx 9.97543 \times 10^{3} \mathrm{~cm}^{-1}, & \text { in medium two. }\end{cases}
$$


The above implies that a coefficient in (1.7) is discontinuous at the lens interface. Relation (1.9) represents a single surface situation. Multiple surface scenarios can also be discussed with additional $\kappa$ values. Needless to say, the discontinuity adds considerable difficulties to the computation of the numerical solution of the differential equation $[3,5,9,15]$.

We employ Neumann boundary conditions

$$
u_{r}(z, 0)=u_{r}\left(z, R_{1}\right)=0, \quad z>0,
$$

at the bottom, $r=0$ and top, $r=R_{1}$ of the rectangular domain.

For the initial solution of boundary value problem (1.7), (1.8), we use the following approximation of a Gaussian beam with point source [4]

$$
u(z, r)=\frac{A}{1+i \vartheta} \exp \left(i k z-\frac{r^{2}}{\beta^{2}(1+i \vartheta)}\right),
$$

where $\beta_{0}$ is the Gaussian beam width, while $\vartheta, \beta$ and $A$ are parameters such that

$$
\vartheta=\frac{2 z}{\beta^{2} k}, \quad \frac{1}{\beta^{2}}=\frac{1}{\beta_{0}^{2}}+\frac{i k}{2 z_{0}}, \quad A=e^{i k z_{0}} .
$$

\section{Base Difference Scheme and Stability}

Let $0 \leq z \leq Z$ for (1.8)-(1.11). Further, let $h=R_{1} / M, \tau=Z / N$, where $M, N \in$ $\mathbb{Z}^{+}$are sufficiently large. We may introduce the uniform grid region,

$$
\Omega_{h, \tau}=\{(m h, n \tau) \mid 0 \leq m \leq M, 0 \leq n \leq N\}
$$

over the rectangular domain $\Omega$ used. For the sake of simplicity, we denote $r_{m}=m h$ and $z_{n}=n \tau$. In addition, we will use $z_{n-\alpha}, 0<\alpha<1$, for specifying a non-grid point between $z_{n-1}$ and $z_{n}$ whenever needed.

Let us start with a linear second-order partial differential equation of the form

$$
c_{5} \frac{\partial^{2} u}{\partial z \partial r}+c_{4} \frac{\partial^{2} u}{\partial r^{2}}+c_{3} \frac{\partial u}{\partial r}+c_{2} \frac{\partial u}{\partial z}+c_{1} u+c_{0}=0, \quad(r, z) \in \Omega
$$

together with (1.10), (1.11). The coefficients $c_{i}$ of (2.1) are functions of $z$ and $r$ and may be discontinuous due to (1.9).

We propose a six-point, two-level Crank-Nicholson type scheme for solving (2.1) and (1.9)-(1.11),

$$
\begin{aligned}
& \frac{c_{5}}{2 h \tau}\left[u_{m+1, n}-u_{m-1, n}-u_{m+1, n-1}+u_{m-1, n-1}\right] \\
& \quad+\frac{c_{4}}{2 h^{2}}\left[u_{m+1, n}-2 u_{m, n}+u_{m-1, n}+u_{m+1, n-1}-2 u_{m, n-1}+u_{m-1, n-1}\right] \\
& \quad+\frac{c_{3}}{4 h}\left[u_{m+1, n}-u_{m-1, n}+u_{m+1, n-1}-u_{m-1, n-1}\right] \\
& \quad+\frac{c_{2}}{\tau}\left[u_{m, n}-u_{m, n-1}\right]+\frac{c_{1}}{2}\left[u_{m, n}+u_{m, n-1}\right]+c_{0}=0
\end{aligned}
$$


The above can be conveniently reformulated to a partial difference equation

$$
\begin{gathered}
\left(\frac{c_{5}}{2 h \tau}+\frac{c_{4}}{2 h^{2}}+\frac{c_{3}}{4 h}\right) u_{m+1, n}+\left(-\frac{c_{4}}{h^{2}}+\frac{c_{2}}{\tau}+\frac{c_{1}}{2}\right) u_{m, n}+\left(-\frac{c_{5}}{2 h \tau}+\frac{c_{4}}{2 h^{2}}-\frac{c_{3}}{4 h}\right) u_{m-1, n} \\
=\left(\frac{c_{5}}{2 h \tau}-\frac{c_{4}}{2 h^{2}}-\frac{c_{3}}{4 h}\right) u_{m+1, n-1}+\left(\frac{c_{4}}{h^{2}}+\frac{c_{2}}{\tau}-\frac{c_{1}}{2}\right) u_{m, n-1} \\
\quad\left(-\frac{c_{5}}{2 h \tau}-\frac{c_{4}}{2 h^{2}}+\frac{c_{3}}{4 h}\right) u_{m-1, n-1}+c_{0} .
\end{gathered}
$$

Let $w$ be a sufficiently smooth function defined on $\Omega$. We define

$$
P w(r, z)=c_{5} \frac{\partial^{2} w}{\partial z \partial r}+c_{4} \frac{\partial^{2} w}{\partial r^{2}}(r, z)+c_{3} \frac{\partial w}{\partial r}(r, z)+c_{2} \frac{\partial w}{\partial z}(r, z)+c_{1} w(r, z)+c_{0}
$$

and

$$
\begin{gathered}
P_{h, \tau} w_{m, n-1 / 2}=\left(\frac{c_{5}}{2 h \tau}+\frac{c_{4}}{2 h^{2}}+\frac{c_{3}}{4 h}\right) w_{m+1, n}+\left(-\frac{c_{4}}{h^{2}}+\frac{c_{2}}{\tau}+\frac{c_{1}}{2}\right) w_{m, n} \\
\quad+\left(-\frac{c_{5}}{2 h \tau}+\frac{c_{4}}{2 h^{2}}-\frac{c_{3}}{4 h}\right) w_{m-1, n}+\left(-\frac{c_{5}}{2 h \tau}+\frac{c_{4}}{2 h^{2}}+\frac{c_{3}}{4 h}\right) w_{m+1, n-1} \\
\quad+\left(-\frac{c_{4}}{h^{2}}-\frac{c_{2}}{\tau}+\frac{c_{1}}{2}\right) w_{m, n-1}+\left(\frac{c_{5}}{2 h \tau}+\frac{c_{4}}{2 h^{2}}-\frac{c_{3}}{4 h}\right) w_{m-1, n-1}+c_{0}
\end{gathered}
$$

By expressing the function $w$ at the grid points as Taylor expansions evaluated at reference point $\left(r_{m}, z_{n-\frac{1}{2}}\right)$ and substituting these into (2.3), it can be shown that

$$
\left\|\left(P-P_{h, \tau}\right) w_{m, n-1 / 2}\right\|=O\left(h^{2}+h^{2} \tau^{2}+\tau^{2}\right) .
$$

Let $\sigma=\tau / h$ be bounded and $\sigma \rightarrow 0$ as $h, \tau \rightarrow 0$. Then (2.4) ensures

1. the consistency of the finite difference scheme (2.2);

2. a second-order local truncation error of the scheme (2.2);

3. the numerical stability depends on the particular coefficients of the differential equation considered.

Definition 2.1. Consider a homogeneous finite difference scheme written as a system of linear equations as below:

$$
B \mathbf{u}_{n}=C \mathbf{u}_{n-1}
$$

or

$$
\mathbf{u}_{n}=B^{-1} C \mathbf{u}_{n-1}
$$

where vector $\mathbf{u}_{n}=\left\{u_{k, n}\right\}_{k=0}^{M}$ and the difference operators $B, C \in \mathbb{C}^{M \times M}$ are coefficient matrices. Let $E=B^{-1} C$. If there exists a constant $K>0$ independent of $n$, $h$ and $\tau$ such that $\left\|E^{n}\right\| \leq K$ for some norm $\|\cdot\|$, we say that the scheme is stable in the Lax-Richtmyer sense [10,11]. 
Due to the inclusion of a cross-derivative term in our transformed equation, our difference scheme will not be stable in the Lax-Richtmyer sense. We define a notion of practical stability which holds for a range of propagation step sizes that afford us sufficient resolution in our simulations.

Definition 2.2. Let $\rho\left(B^{-1} C\right)$ be the spectral radius of kernel matrix $B^{-1} C$. If

$$
\rho\left(B^{-1} C\right) \leq 1
$$

at all propagation steps $0<n \tau<T$ with a transverse direction step size $\epsilon_{0}<h<\epsilon_{1}$ for some $\epsilon_{1}>\epsilon_{0}>0$, we say that the scheme

$$
B \mathbf{u}_{n}=C \mathbf{u}_{n-1}
$$

is stable within a parameter range.

While this stability condition does not specify a norm for convergence, it does guarantee that perturbations will not increase exponentially with $n$.

Definition 2.3. A matrix $A \in \mathbb{C}^{n \times n}$ is said to be positive semistable if every eigenvalue of $A$ has nonnegative real part.

Theorem 2.4. Let $A, B, C, G \in \mathbb{C}^{M \times M}$ be such that

$$
B=G+A, \quad C=G-A
$$

Then the difference scheme defined by

$$
B \mathbf{u}_{n}=C \mathbf{u}_{n-1}
$$

is stable if and only if $G^{-1} A$ is positive semistable.

Corollary 2.5. Let $A, B, C \in \mathbb{C}^{M \times M}$, $d$ be a positive real number such that

$$
B=d I+A, \quad C=d I-A .
$$

Then the difference scheme defined by

$$
B \mathbf{u}_{n}=C \mathbf{u}_{n-1}
$$

is stable if and only if $A$ is positive semistable.

Recall (1.8). We have the corresponding paraxial Helmholtz coefficients for the general equations (2.1) and (2.2),

$$
c_{5}=0, c_{4}=1, c_{3}=\frac{1}{r}, c_{2}=-2 i \kappa, c_{1}=0, c_{0}=0 .
$$


It follows therefore that (2.2), (1.10) and (1.11) can be simplified to the following homogeneous paraxial Helmholtz difference scheme,

$$
\begin{aligned}
& -\alpha\left(1+\frac{1}{2 m}\right) u_{m+1, n}+(2+2 \alpha) u_{m, n}-\alpha\left(1-\frac{1}{2 m}\right) u_{m-1, n} \\
& \quad=\alpha\left(1+\frac{1}{2 m}\right) u_{m+1, n-1}+(2-2 \alpha) u_{m, n-1}+\alpha\left(1-\frac{1}{2 m}\right) u_{m-1, n-1}, \\
& u_{m, 0}=e^{-h m / \beta_{0}} \\
& -2 \alpha u_{1, n}+(2+2 \alpha) u_{0, n}=2 \alpha u_{1, n-1}+(2-2 \alpha) u_{0, n-1} \\
& (2+2 \alpha) u_{M, n}-2 \alpha u_{M-1, n}=(2-2 \alpha) u_{M, n-1}+2 \alpha u_{M-1, n-1}
\end{aligned}
$$

where

$$
\alpha=-\frac{\tau i}{2 \kappa h^{2}} .
$$

Following the analysis method outlined above, we can express our scheme in matrix form

$$
B \mathbf{u}_{n}=C \mathbf{u}_{n-1}
$$

where $B=G+A, C=G-A, G=2 I$, and $A$ is tridiagonal. Investigating properties of the eigenvalues of $A=\left\{a_{m, n}\right\}$, where

$$
\begin{aligned}
a_{m, m} & =2 \alpha, \quad m=0,1, \ldots, M, \\
a_{m, m-1} & =-\alpha\left(1-\frac{1}{2 m}\right), \quad m=1,2, \ldots, M-1, \\
a_{M, M-1} & =-2 \alpha, \\
a_{m, m+1} & =-\alpha\left(1+\frac{1}{2 m}\right), \quad m=1,2, \ldots, M-1, \\
a_{0,1} & =-2 \alpha,
\end{aligned}
$$

w are able to show that the eigenvalues of matrix $A$ are purely imaginary, and thus have nonnegative real parts. Thus, $A$ is positive semidefinite. By Corollary 2.5, we can show the following.

Theorem 2.6. Let $\kappa$ be a constant. Then the homogeneous paraxial Helmholtz difference scheme (2.6)-(2.9) is stable. Further, there is lower boundary restriction on the step size parameter, $h$, in this case.

\section{3. $z$-Stretching Domain Transformation}

One possible way of avoiding the computational difficulties presented by the discontinuity of $\kappa$ at the interface is by decomposing the domain into three sections, 
pre-lens, lens, and post-lens, and stretching each segment by one-to-one transformations onto rectangular areas. We would then be able to use conventional finite difference techniques, such as that introduced in Section 2, to solve (2.1) together with initial-boundary conditions on each segment. The grid stretch can be achieved either in the direction of electro-magnetic wave propagation $z$, or the direction of $r$. Each of the approaches have distinct advantages. We will only focus on the former strategy in this paper. In this case, the numerical solution computed at the rightmost edge of the pre-lens segment becomes the initial condition of the next segment.

Let $r=r(\xi, \zeta), z=z(\xi, \zeta)$ be the one-to-one stretching transformation to be used. Thus,

$$
\begin{aligned}
& \frac{\partial u}{\partial r}=\frac{\partial u}{\partial \xi} \frac{\partial \xi}{\partial r}+\frac{\partial u}{\partial \zeta} \frac{\partial \zeta}{\partial r}, \quad \frac{\partial u}{\partial z}=\frac{\partial u}{\partial \xi} \frac{\partial \xi}{\partial z}+\frac{\partial u}{\partial \zeta} \frac{\partial \zeta}{\partial z} \\
& \frac{\partial^{2} u}{\partial r^{2}}=\frac{\partial u}{\partial \xi} \frac{\partial^{2} \xi}{\partial r^{2}}+\frac{\partial^{2} u}{\partial \xi^{2}}\left(\frac{\partial \xi}{\partial r}\right)^{2}+2 \frac{\partial^{2} u}{\partial \xi \partial \zeta} \frac{\partial \xi}{\partial r} \frac{\partial \zeta}{\partial r}+\frac{\partial u}{\partial \zeta} \frac{\partial^{2} \zeta}{\partial r^{2}}+\frac{\partial^{2} u}{\partial \zeta^{2}}\left(\frac{\partial \zeta}{\partial r}\right)^{2} .
\end{aligned}
$$

A substitution of the above into (1.8) yields

$$
\begin{gathered}
2 i \kappa\left(\frac{\partial u}{\partial \xi} \frac{\partial \xi}{\partial z}+\frac{\partial u}{\partial \zeta} \frac{\partial \zeta}{\partial z}\right)=\frac{\partial u}{\partial \xi} \frac{\partial^{2} \xi}{\partial r^{2}}+\frac{\partial^{2} u}{\partial \xi^{2}}\left(\frac{\partial \xi}{\partial r}\right)^{2}+2 \frac{\partial^{2} u}{\partial \xi \partial \zeta} \frac{\partial \xi}{\partial r} \frac{\partial \zeta}{\partial r} \\
+\frac{\partial u}{\partial \zeta} \frac{\partial^{2} \zeta}{\partial r^{2}}+\frac{\partial^{2} u}{\partial \zeta^{2}}\left(\frac{\partial \zeta}{\partial r}\right)^{2}+\frac{1}{r}\left(\frac{\partial u}{\partial \xi} \frac{\partial \xi}{\partial r}+\frac{\partial u}{\partial \zeta} \frac{\partial \zeta}{\partial r}\right),
\end{gathered}
$$

which can be regrouped into

$$
\begin{gathered}
\left(2 i \kappa \frac{\partial \zeta}{\partial z}-\frac{\partial^{2} \zeta}{\partial r^{2}}-\frac{1}{r} \frac{\partial \zeta}{\partial r}\right) \frac{\partial u}{\partial \zeta}=\left(-2 i \kappa \frac{\partial \xi}{\partial z}+\frac{\partial^{2} \xi}{\partial r^{2}}+\frac{1}{r} \frac{\partial \xi}{\partial r}\right) \frac{\partial u}{\partial \xi} \\
+\left(\frac{\partial \xi}{\partial r}\right)^{2} \frac{\partial^{2} u}{\partial \xi^{2}}+2\left(\frac{\partial \xi}{\partial r} \frac{\partial \zeta}{\partial r}\right) \frac{\partial^{2} u}{\partial \xi \partial \zeta}+\left(\frac{\partial \zeta}{\partial r}\right)^{2} \frac{\partial^{2} u}{\partial \zeta^{2}}
\end{gathered}
$$

To map the lens area $\Omega=\left\{0 \leq z \leq Z,(z-R)^{2}+r^{2} \leq R^{2}, r \geq 0\right\}$ into a rectangular area $\tilde{\Omega}=\left\{0 \leq \zeta \leq Z, 0 \leq \xi \leq R_{1}\right\}$, a natural choice is the following transformation,

$$
\xi(r, z)=r, \quad \zeta(r, z)=\frac{z-R+\sqrt{R^{2}-r^{2}}}{Z-R+\sqrt{R^{2}-r^{2}}} Z .
$$

In this particular case we have

$$
\begin{aligned}
& \frac{\partial \xi}{\partial r}=1, \quad \frac{\partial \xi}{\partial z}=0, \quad \frac{\partial^{2} \xi}{\partial r^{2}}=0 \\
& \frac{\partial \zeta}{\partial r}=\frac{r Z(z-Z)}{\rho^{2} \sqrt{R^{2}-r^{2}}}, \quad \frac{\partial \zeta}{\partial z}=\frac{Z}{\rho}, \frac{\partial^{2} \zeta}{\partial r^{2}}=\frac{Z(z-Z)\left(\rho R^{2}+2 r^{2} \sqrt{R^{2}-r^{2}}\right)}{\rho^{3}\left(R^{2}-r^{2}\right)^{3 / 2}},
\end{aligned}
$$


where $\rho=Z-R+\sqrt{R^{2}-r^{2}}$. We define functions

$$
\begin{aligned}
\phi(\xi, \zeta) & :=\frac{\partial \zeta}{\partial r}(r(\xi, \zeta), z(\xi, \zeta))=\frac{(\zeta-Z) \xi}{\sqrt{R^{2}-\xi^{2}}\left[Z-\left(R-\sqrt{R^{2}-\xi^{2}}\right)\right]} \\
\psi(\xi, \zeta) & :=\frac{\partial^{2} \zeta}{\partial r^{2}}(r(\xi, \zeta), z(\xi, \zeta))=\frac{(\zeta-Z)\left[R^{3}-\sqrt{R^{2}-\xi^{2}}\left(R^{2}+2 \xi^{2}\right)-Z\right]}{\left(R^{2}-\xi^{2}\right)^{\frac{3}{2}}\left[Z-\left(R-\sqrt{R^{2}-\xi^{2}}\right)\right]^{2}}, \\
\theta(\xi, \zeta) & :=\frac{\partial \zeta}{\partial z}(r(\xi, \zeta), z(\xi, \zeta))=\frac{Z}{Z-\left(R-\sqrt{R^{2}-\xi^{2}}\right)} .
\end{aligned}
$$

Subsequently, (3.1) can be simplified to

$$
\left(2 i \kappa \theta-\psi-\frac{1}{\xi} \phi\right) \frac{\partial u}{\partial \zeta}=\frac{1}{\xi} \frac{\partial u}{\partial \xi}+\frac{\partial^{2} u}{\partial \xi^{2}}+2 \phi \frac{\partial^{2} u}{\partial \xi \partial \zeta}+\phi^{2} \frac{\partial^{2} u}{\partial \zeta^{2}} .
$$

It can be demonstrated that at every point in the transformed lens segment, we have

$$
\phi^{2}\left|\frac{\partial^{2} u}{\partial \zeta^{2}}\right| \leq \frac{R_{1}^{2} Z}{\left(R^{2}-R_{1}^{2}\right)\left[Z-\left(R-\sqrt{R^{2}-R_{1}^{2}}\right)\right]}\left|\frac{\partial^{2} u}{\partial z^{2}}\right| \approx 0 .
$$

Thus for a typical lens where the slowly varying envelope approximation is applicable, (3.2) can be simplified to a more appropriate form for computations within the transformed lens area,

$$
\left(2 i \kappa \theta-\psi-\frac{1}{\xi} \phi\right) \frac{\partial u}{\partial \zeta}=\frac{1}{\xi} \frac{\partial u}{\partial \xi}+\frac{\partial^{2} u}{\partial \xi^{2}}+2 \phi \frac{\partial^{2} u}{\partial \xi \partial \zeta} .
$$
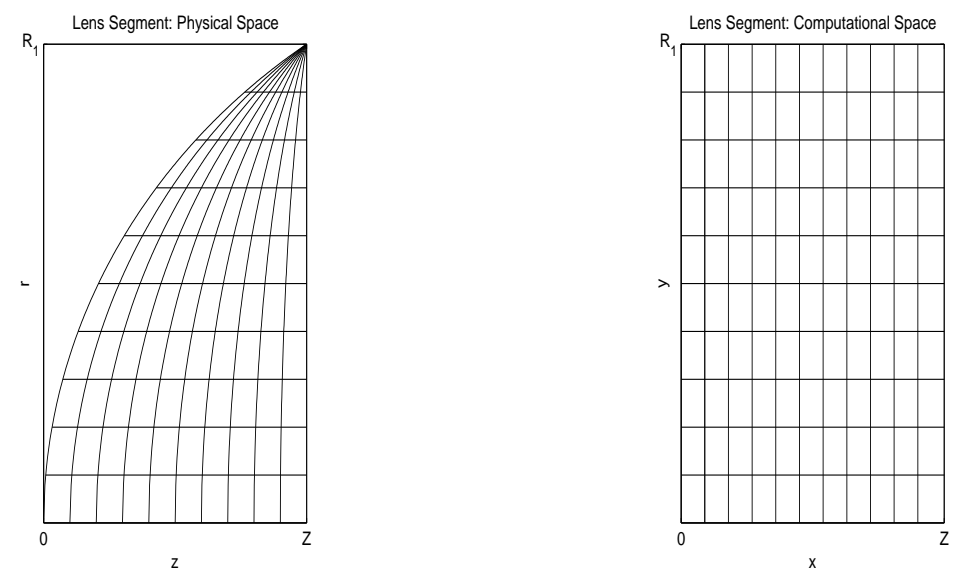

FiguRE 3.1. LEFT: An in-lens domain before a $z$-stretching. RIGHT: The in-lens domain after a $z$-stretching. 
In Figure 3.1, we illustrate the $z$-stretching by showing an example of the grid within the lens before and after the stretching. It is evident that the transformation enables the six-point scheme to work accurately and efficiently. Instead of (2.5), now we have the following set of adjusted coefficients for the in-lens segment difference scheme,

$$
c_{5}=2 \phi, c_{4}=1, c_{3}=\frac{1}{\xi}, c_{2}=-2 i \kappa \theta+\psi+\frac{1}{\xi} \phi, c_{1}=0, c_{0}=0 .
$$

Subsequently, (2.6) can be modified to

$$
\begin{aligned}
& -\gamma\left[\frac{2 \phi}{h}+\alpha\left(1+\frac{1}{2 m}\right)\right] u_{m+1, n}+(2+2 \alpha \gamma) u_{m, n}-\gamma\left[-\frac{2 \phi}{h}+\alpha\left(1-\frac{1}{2 m}\right)\right] u_{m-1, n} \\
& =\gamma\left[-\frac{2 \phi}{h}+\alpha\left(1+\frac{1}{2 m}\right)\right] u_{m+1, n-1}+(2-2 \alpha \gamma) u_{m, n-1} \\
& \quad+\gamma\left[\frac{2 \phi}{h}+\alpha\left(1-\frac{1}{2 m}\right)\right] u_{m-1, n-1}
\end{aligned}
$$

where

$$
\alpha=\frac{\tau}{h^{2}} \text { and } \gamma=\gamma(\xi, \zeta)=\left(2 i \kappa \theta-\psi-\frac{1}{\xi} \phi\right)^{-1} .
$$

In this method, the Gaussian beam input equation is evaluated at the lens surface, and becomes the initial solution of the simulation at the edge of the lens segment. No computation is necessary in the pre-lens segment. The solution at the right edge of the lens segment becomes the initial solution of the post-lens segment, which we simulate using the homogeneous scheme described earlier.

To determine the boundary conditions applicable within the lens segment, note that

$$
\frac{\partial u}{\partial r}(\xi, \zeta)=\frac{\partial u}{\partial \xi}(\xi, \zeta) \frac{\partial \xi}{\partial r}(\xi, \zeta)+\frac{\partial u}{\partial \zeta}(\xi, \zeta) \frac{\partial \zeta}{\partial r}(\xi, \zeta)
$$

thus

$$
\frac{\partial u}{\partial \xi}(0, \zeta)=\frac{\partial u}{\partial \xi}\left(R_{1}, \zeta\right)=0, \quad 0<\zeta<Z
$$

It is interesting to note that for a lens that tapers to a point at the top, the geometric interpretation of this new boundary condition is that the single point $\left(R_{1}, Z\right)$ has been stretched into the upper edge of our transformed rectangular domain, i.e. the upper boundary in the computational space corresponds to the single point $\left(R_{1}, Z\right)$ in the physical space.

To demonstrate stability, we use the matrix analysis method introduced in the previous section. For our scheme $B \mathbf{u}_{n}=C \mathbf{u}_{n-1}$, we have $B=G+A, C=G-A$ 
with matrix $G=\left\{g_{m, n}\right\}$ and $A=\left\{a_{m, n}\right\}$, where

$$
\begin{aligned}
& g_{m, m}=2, \quad m=0,1, \ldots, M, \\
& g_{m, m-1}=\frac{2 \gamma \phi}{h}, \quad m=1,2, \ldots, M-1, \\
& g_{M, M-1}=0, \\
& g_{m, m+1}=\frac{-2 \gamma \phi}{h}, \quad m=1,2, \ldots, M-1, \\
& g_{0,1}=0, \\
& a_{m, m}= 2 \alpha \gamma, \quad m=0,1, \ldots, M, \\
& a_{m, m-1}=-\alpha \gamma\left(1-\frac{1}{2 m}\right), \quad m=1,2, \ldots, M-1, \\
& a_{M, M-1}=-2 \alpha \gamma, \\
& a_{m, m+1}=-\alpha \gamma\left(1+\frac{1}{2 m}\right), \quad m=1,2, \ldots, M-1, \\
& a_{0,1}=-2 \alpha \gamma .
\end{aligned}
$$

Examining the real part of function $\gamma(\xi, \zeta)$ resulting from our chosen transformation, we see that matrix $A$ is positive semistable. This property will still hold if the transformation is adapted to other convex lens shapes. Further, if we have a transverse step size $h$ such that

$$
h>2|\gamma(\xi, \zeta) \phi(\xi, \zeta)| \text { for } 0 \leq \xi \leq R_{1}, 0 \leq \zeta \leq Z
$$

or equivalently, if the number of grid points in the $\xi$ direction, $M$, is such that

$$
M<\frac{R_{1}}{h_{\min }}
$$

where

$$
h_{\text {min }}=2 \max _{\xi, \zeta}|\gamma(\xi, \zeta) \phi(\xi, \zeta)|
$$

then matrix $G+G^{*}$ is positive definite. Then based on Theorem (2.4), we can prove the following.

Theorem 3.1. Let $\kappa$ be discontinuous as given by (1.9) and

$$
h>2|\gamma(\xi, \zeta) \phi(\xi, \zeta)| \text { for } 0 \leq \xi \leq R_{1}, 0 \leq \zeta \leq Z .
$$

Then the difference scheme (3.4)-(3.5) is stable on z-stretched domains.

For the parameter values utilized in our simulations

$$
\kappa=9.97543 \times 10^{3}, \quad R=1.969, \quad Z=0.7643, \quad R_{1}=1.5574
$$

we need $M \leq 1.2092 \times 10^{4}$. We list a sampling of maximum grid points $M$ for other parameter values. 


\begin{tabular}{|c|ccc|}
\hline$Z$ & $k=8000$ & $k=10000$ & $k=12000$ \\
\hline 0.1 & 7439 & 9365 & 11279 \\
0.3 & 5642 & 7046 & 8848 \\
0.5 & 4059 & 5064 & 6068 \\
0.7 & 2496 & 3104 & 3711 \\
0.9 & 1033 & 1257 & 1479 \\
\hline
\end{tabular}

TABLE 3.1. Maximum grid points in the transverse direction with $R=1$.

\section{Simulation Results and Observations}

All simulated results were implemented on dual processor DeLL workstations with at least double precision. MATLAB, ForTRAN and $\mathrm{C}++$ programming languages were utilized. Dimensionless models are used throughout the computations. For the sake of simplicity, we do not tend to re-scale numerical solutions back to their original physical dimensions in simulations.

In the following numerical experiments we use parameters (3.6) listed in the previous section. We further select $h=R_{1} / M$ where $M=5 \times 10^{3}$ and $\tau=Z / N$ where $N=1.6 \times 10^{4}$ in the six-point scheme used in the computational solution space after a designated $z$-stretching. 


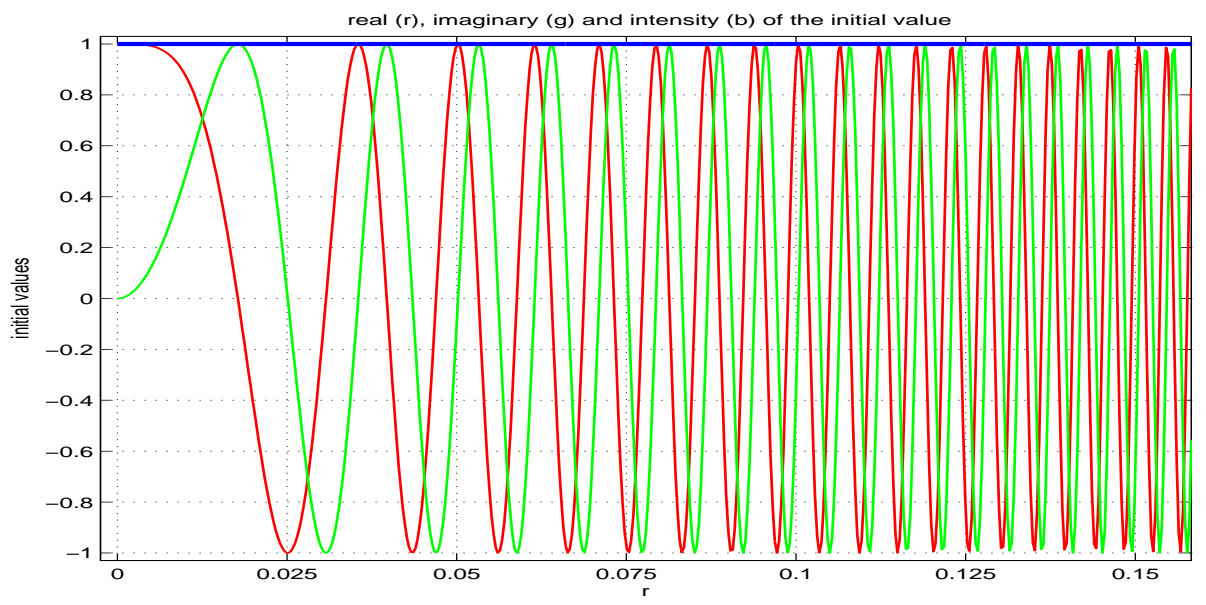

FiguRE 4.1. Normalized initial value function $u(r, 0)$. Red curve is for the real part and green curve is for the imaginary part of the function. Highly oscillatory features of the function is clear.

We show the real part of the simulated solution, $a(z)=\operatorname{real}\{u(0, z)\}$, in Figures 4.2-3. We may observe that while the function value of $a$ is relatively stable before the focusing point $z_{f} \approx 0.94778$, it increases dramatically as $z \rightarrow z_{f}$. This can be viewed more precisely in the enlarged picture of Figure 4.3.

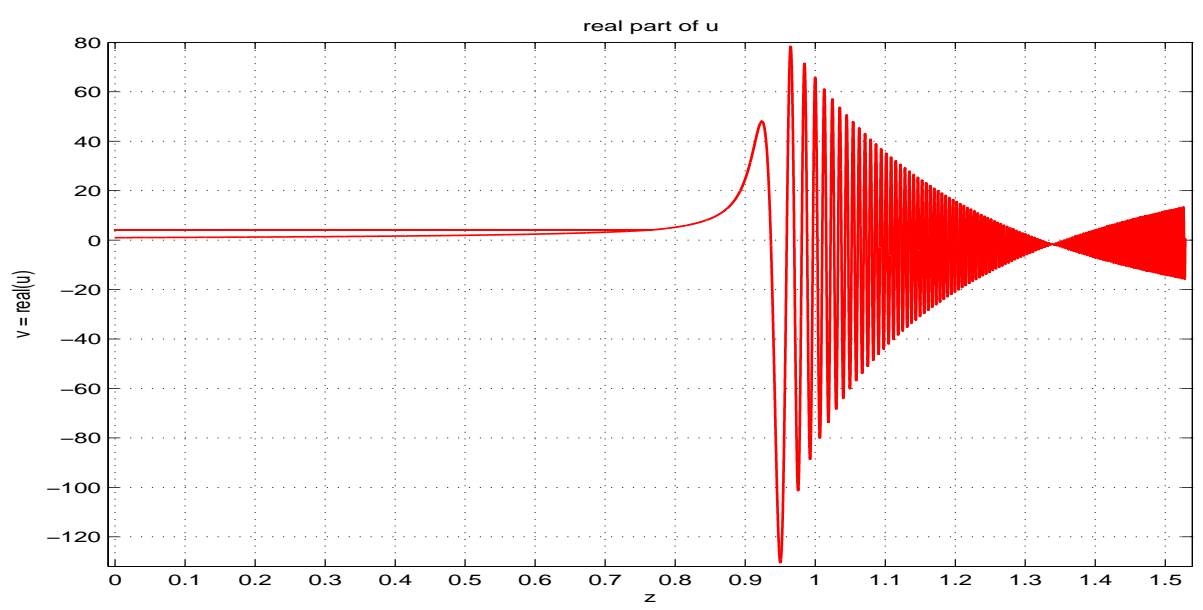

FiguRE 4.2. Real part of the simulated solution at the center point $r=0$. The numerical solution increases rapidly as $z$ approaches the focusing location. Then the simulated oscillatory wave diffuses after the focusing point. 


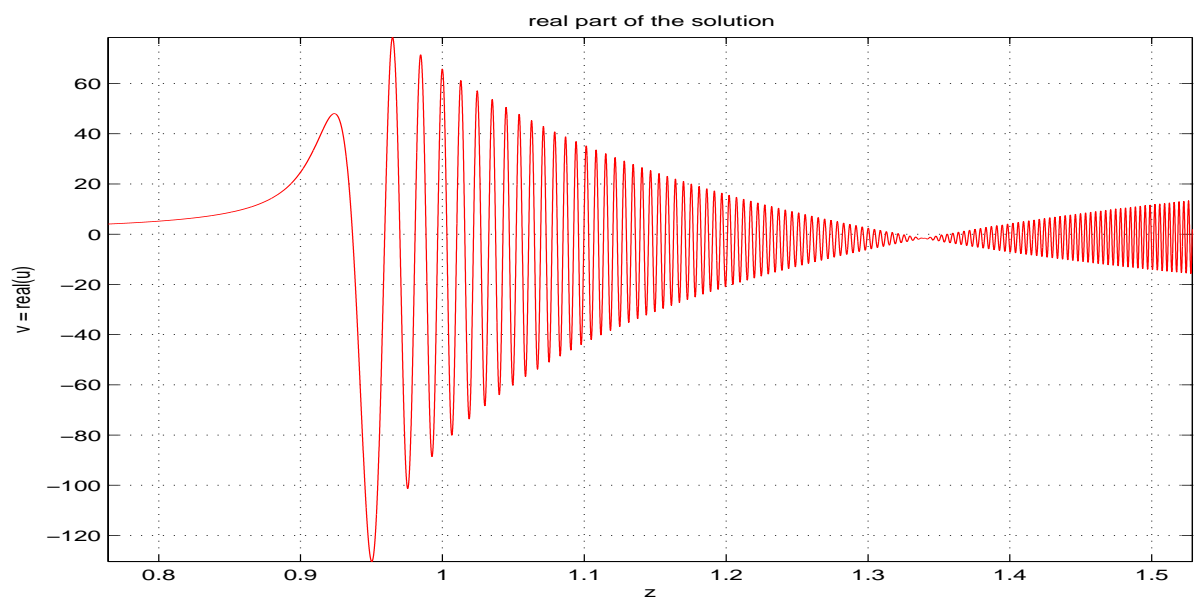

FiguRE 4.3. More detailed real part of the simulated solution near the focus point, $r=0$. Same conditions as in Figure 4.2 are used.

Figures 4.4-5 are devoted to the imaginary part of the numerical solution, $b(z)=$ imaginary $\{u(0, z)\}$. Similar to the real part, $b$ is relatively stable before the focusing point $z_{f} \approx 2.7431$ and is highly oscillatory as $z \rightarrow z_{f}$. The phenomenon can be viewed more clearly in the enlarged picture of Figure 4.5.

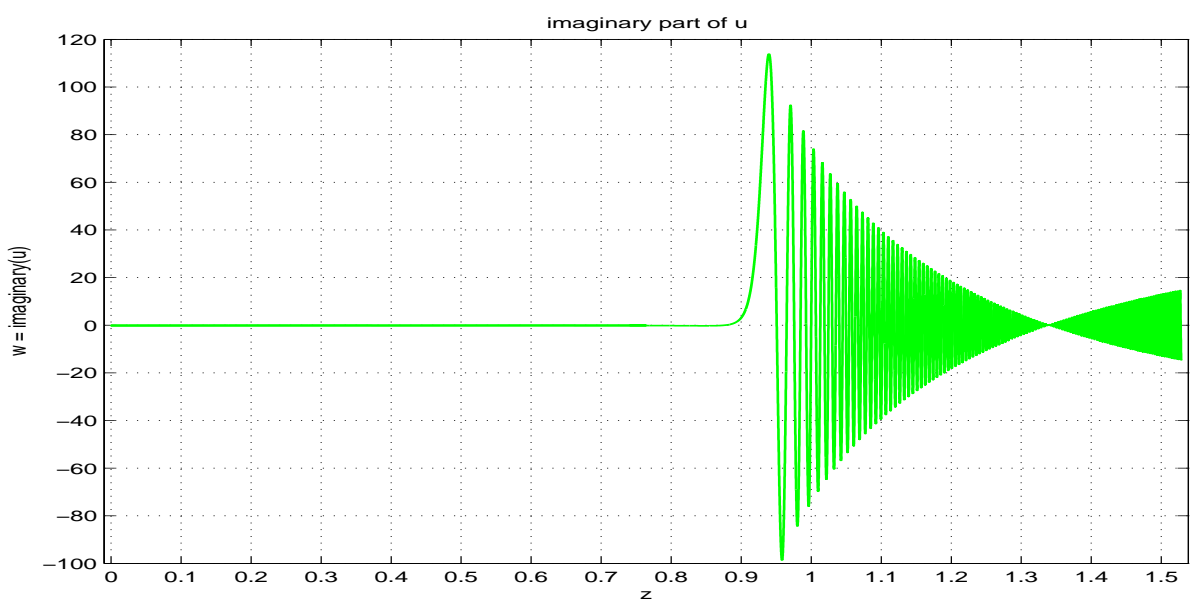

Figure 4.4. Imaginary part of the simulated solution at the center point $r=0$. The numerical solution increases rapidly as $z$ approaches the focusing location. Then the simulated oscillatory wave diffuses after the focusing point. 


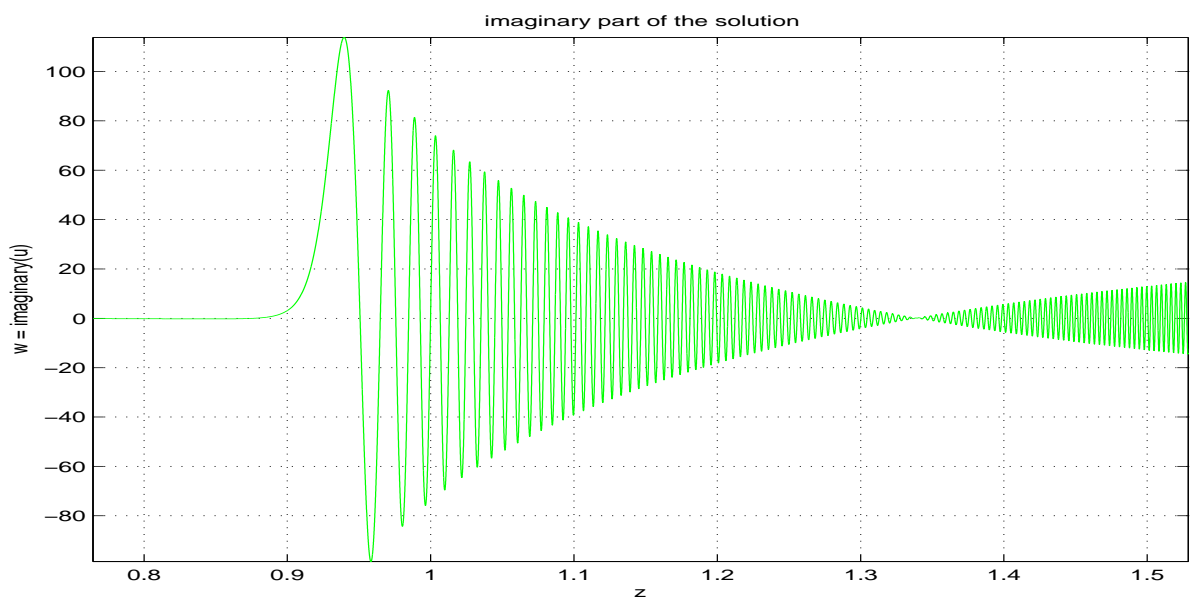

FiguRE 4.5. More detailed imaginary part of the simulated solution near the focus point, $r=0$. Same conditions as in Figure 4.4 are used.

Define the numerical intensity function as

$$
\mathcal{T}(r, z)=\sqrt{\operatorname{real}^{2}[u(r, z)]+\operatorname{imag}^{2}[u(r, z)]} \geq 0, \quad 0 \leq r \leq R_{1}, \quad 0 \leq z \leq Z .
$$

In Figures 4.6-7 we plot this intensity function against the propagation direction $z$ as $r$ being chosen as zero. It is interesting to find that the intensity increases rapidly as $z \rightarrow z_{f}$. The observation is consistent with our previous results.

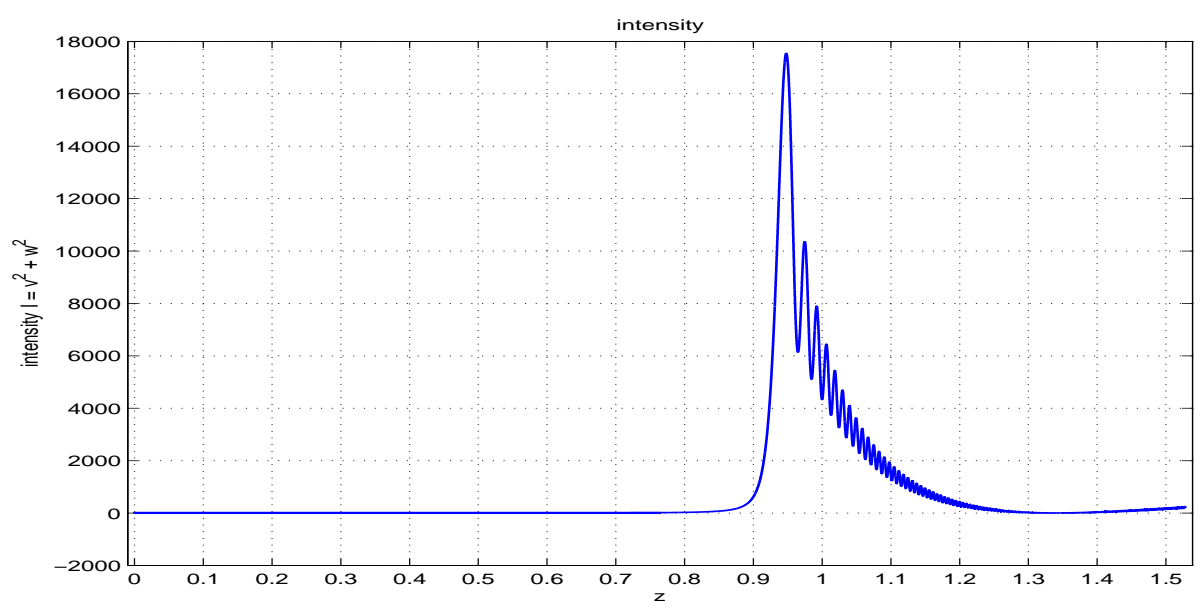

FigURE 4.6. Numerical intensity function of the simulated solution at the center point $r=0$. The intensity increases rapidly as $z$ approaches the focusing location. Then the intensity value diffuses out after the focusing point. 


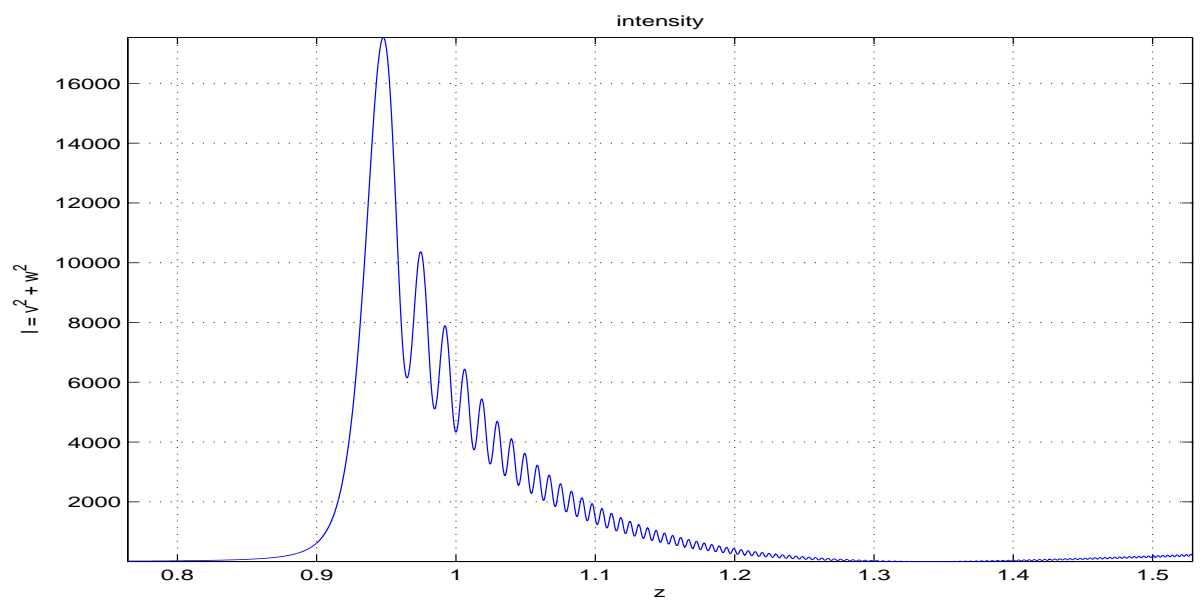

Figure 4.7. More detailed numerical intensity function of the simulated solution near the focus point $(r=0)$. Same conditions as in Figure 4.6 are used.

As a comparison, we further plot the numerical intensity function at the locations near and at the focusing point in Figure 4.8. It is observed that the numerical estimate of the intensity oscillates rapidly in the $r$-direction. The intensity increases sharply near the center point of the lens, $r=0$, while $z$ approaches the focusing point location. The simulated wave profiles well match the experimental results. The algorithms can be used to provide reference values for further explorations.

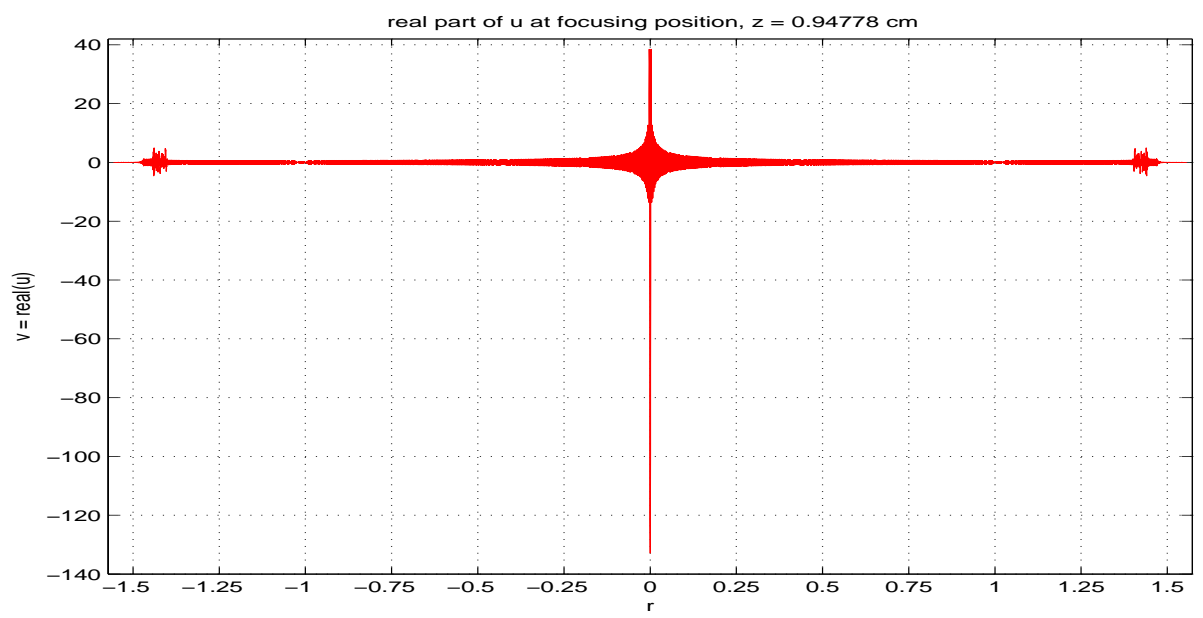

FIGURE 4.8. (ask authors for the image) Highly oscillatory numerical intensity function of the simulated solution at the focusing location $z=2.7431$. The computed intensity increases exponentially near the center, as compared with much lower profiles away from the focusing area.

\section{Conclusions}


In this discussion, we have employed a z-stretching domain transformation to map the lens and post-lens domains into convenient rectangular shapes, where we can utilize well established finite difference methods on uniform grids. The resulting method provides a useful approximation technique that can be efficiently implemented with less than 50 lines of Matlab code in the simulation loops.

A more powerful strategy may be the use of optimally combined $z$ and $r$ stretching transformations to increase computational resolution and accuracy of the numerical solution in critical local regions. Magnifying a particular subregion in the transformed coordinate space is computationally equivalent to increasing the refinement of the grid in that region using techniques such as adaptive mesh refinement $[2,14]$. Alternatively, domain transformation could facilitate the use of established adaptive grid techniques, since applications of standard mesh refinement or moving mesh technics are straightforward on rectangular domains [8, 14]. Further, hyperbolic smoothness maps have also been proved to be extremely useful auxiliary tools to consider in practical optical beam computations [15]. Detailed discussions will be given in our forthcoming reports.

\section{References}

[1] Y. B. Band, Light and Matter: Electromagnetism, Optics, Spectroscopy and Lasers, John Wiley \& Sons, West Sussex, 2006.

[2] M. Berger, J. Oliger, Adaptive mesh refinement for hyperbolic partial differential equations, J. Comp. Phys., 53 (1984) 484-512.

[3] H. Cheng, P. Lin, Q. Sheng and R. Tan, Solving degenerate reaction-diffusion equations via variable step Peaceman-Rachford splitting, SIAM J. Sci. Comput., 25 (2003) 1273-1292.

[4] H. J. Eom, Electromagnetic Wave Theory for Boundary-Value Problems - An Advanced Course on Analytical Methods, Springer-Verlag, Berlin Heidelberg, 2004.

[5] J. W. Goodman, Introduction to Fourier Optics, Third Edition, Roberts \& Company Publishers, Denver, 2004.

[6] S. Guha, Validity of the paraxial approximation in the focal region of a smallf-number lens, Optical Lett., 26 (2001), 1598-1600.

[7] S. Guha and G. D. Gillen, Description of light propagation through a circular aperture using non-paraxial vector diffraction theory, Optics Express, 13 (2005), 1424-1447. 
[8] I. S. Kim and W. J. R. Hoefer, A local mesh refinement algorithm for the time domain-finite difference method using Maxwell's equations, IEEE Trans. Microwave Theory \& Tech., 38 (1990), 812-815.

[9] E. Larsson, A domain decomposition method for the Helmholtz equation in a multilayer domain, SIAM J. Sci. Comput., 20 (1999), 1713-1731.

[10] P. D. Lax and R. D. Richtmeyer, Survey of the stability of linear finite difference equations, Comm. Pure Appl. Math, 9 (1956), 267-294.

[11] K. W. Morton, Stability of finite difference approximations to a diffusionconvection equation, Int. J. Num. Meth. Eng., 15 (1980), 677-683.

[12] T. Poon and T. Kim, Engineering Optics with Matlab, World Scientific Publishing, Singapore, 2006.

[13] B. E. A. Saleh and M. C. Teich, Fundamentals of Photonics, John Wiley \& Sons, New York, 1991.

[14] Q. Sheng and H. Cheng, An adaptive grid method for degenerate semilinear quenching problems, Computers Math. Appl., 39 (2000), 57-71.

[15] Q. Sheng, S. Guha and L. Gonzalez, Fast computer simulations of the Helmholtz equation solutions in multi-layer medians with high wavenumbers, Technical Report, AFRL/MLPJ-0804 (2006).

[16] J. Shibayama, K. Matsubara M. Sekiguchi, J. Yamauchi and H. Nakano, Efficient nonuniform schemes for paraxial and wide-angle finite-difference beam propagation methods, J. Lightwave. Tech., 17 (1999), 677-683. 